\title{
Clinical, biochemical, and epidemiological characteristics of patients infected with Histoplasma capsulatum in a non-endemic area of Mexico
}

\begin{abstract}
Introduction: Histoplasmosis is the most prevalent systemic mycosis in Mexico. However, since 1995, the routine registry of this disease was interrupted in the country; therefore, the current magnitude of the disease is unknown. This study aimedto describe the epidemiological, clinical, and paraclinical characteristics of the histoplasmosis infectionwhich has a wide variety of clinical manifestations - in the population that received care in Mexico's National Institute for Respiratory Diseases.
\end{abstract}

Material and methods: This study used a retrospective design, and the analyzed data were collected from January 2013 to December 2016.We conducted a comparison of characteristics between patients with pulmonary or disseminated forms of histoplasmosis through $\chi 2$, Student's T, or Mann-Whitney U tests.

Results: In total, 61 patients were included. The average age was 36.1 years $\pm 12.4 ; 53$ $(87 \%)$ were men, and the male-female ratio was $6: 1$. Moreover, $80 \%$ of the patients had immunosuppression; $94 \%$ had HIV; $50.8 \%$ had disseminated histoplasmosis, so it had the most common presentation; and 57\% had anemia (i.e. median $\mathrm{Hb} 10 \mathrm{~g} / \mathrm{dL}$ ). Of the 118 fungus cultures, $74 \%$ (87) reported growth. The Histoplasma antigen was positive in $67 \%$ $(14 / 21)$ of the cases, andmortality rate was estimated at $7 \%$.

Conclusions: Compared with data published in 1995, we observed a change in the epidemiology and risk factors of histoplasmosis; specifically, there was a lower proportion of occupational exposure and an increase in the number of cases in non-endemic areas (i.e. low risk of transmission). Our results generally corroborate the literature, suggesting that the acquired immunodeficiency syndrome (AIDS) pandemic, the use of immunosuppressant therapy, and the rise in the number of transplants have increased the overall risk of histoplasmosis, even in areas that were not previously identified as endemic. Generally, our studyhighlights the importance of routine registry of this disease in Mexico.
Volume 8 Issue 4 - 2020

\author{
Eduardo Becerril Vargas, Ángel Sánchez \\ Tinajero, José Arturo Martínez Orozco, \\ Abraham Onel Pacheco, Danna Patricia Ruíz \\ Santillán, Fernando Rosalio Morales Villareal, \\ Cecilia Iturria Rosales, Roxana Rodríguez \\ Romo,Andrea Iraís Delgado Cueva, Edgar \\ Ricardo Ramírez Estrada \\ National Institute of Respiratory Diseases (INER), Mexico
}

Correspondence: Ángel Sánchez Tinajero, Surgeon, prosgraduate in chemical-biological sciences, National Institute of Respiratory Diseases (INER), Calzada de Tlalpan 4502, I 4080 Mexico City, Mexico, Email angelsan I I @hotmail.com

Received: September 28, 2020 | Published: October 16, 2020

Keywords: endemic, histoplasmosis, nodules, pulmonary, mycosis

\section{Introduction}

Histoplasmosis is an infectious disease caused by Histoplasma capsulatum, a dimorphic fungus associated with high rates of morbidity and mortality. This fungus can be found, for example, in ground that contains decaying guano from birds and bats and in temperate climate zones located between $45^{\circ} \mathrm{N}$ to $30^{\circ} \mathrm{S}$ latitudes. For it to grow, the Histoplasma requires high nitrogen concentrations, an acid medium, and a humid environment. ${ }^{1}$ Traditionally, activities associated with exposure to Histoplasma include mining, construction, demolition, remodeling, woodcutting, and wood collection, all of which increase the chance for the release of infecting conidia that may be inhaled. After inhalation, the lung is the main and recovery starts. Predominantly, primary infections by the Histoplasma capsulatum are asymptomatic, and when it presents symptoms, they are oftenmild respiratory manifestations that resolve spontaneously. Still, this is the entryway for the Histoplasma capsulatum, where pneumonitis is developed. Althoughmacrophages phagocytize the infecting particles, they are often unable to destroy the fungus, thereby allowing it todevelop, intracellularly, to its yeast form. Initially after the infection, the fungus disseminates widely throughout the entire body, but once T Cell-mediated immunity becomespresent, the process stops; however, in patients exposed to high levels of inoculum, symptomatic pulmonary infection is more common andmanyof these patients require hospitalization. ${ }^{2,3}$

Progressive disseminated forms of Histoplasma capsulatum are more frequently observed in pediatric patients younger than 2 years of age and male adults of 54 years of age or older. Moreover, among these populations, chronic disseminated histoplasmosis can present itself even in the absence of a clear history of immunodeficiency. Additionally, persons with different levels of $\mathrm{T}$ Cell-mediated immunity compromise are prone to present severer forms of disseminated histoplasmosis; namely, its course can be more acute and severe among different peoplebecause it is directly related topatients' immunity system deterioration. The most common cause of progressive disseminated histoplasmosis is the presence of an advanced Human Immunodeficiency Virus (HIV) infection, followed by lymphatic leukemia, lymphomas, treatments with high doses of corticosteroids for long periods, being solid organ transplant receptors with chronic rejection, being patients with autoimmune diseases, Sweet syndrome, and treatment with monoclonal antibodies against tumor necrosis factor. ${ }^{2}$ Histoplasmosis is considered to be the most prevalent systemic mycosis worldwide. It is endemic to the American continent and to the USA, with the highest prevalencebeing reported in the Ohio and Mississippi areas. In Europe, cases are rare. Recent 
studies have shown that histoplasmosis is much more disseminated than researchers had previously anticipated, and that the general knowledge aboutits spread is incomplete. Furthermore, the AIDS pandemic, the use of new immunosuppressant therapies, and a higher prevalence of solid organ transplant receptors has increased the risk of histoplasmosis globally, to the point where areas which were previously unidentified as endemic to the fungus have become endemic. ${ }^{4-6}$

Particularly, in 1943, the first case of histoplasmosis was reported in Mexico. Historically, it became known as an occupational disease, being related to miners, farmers, and guano collectors. From 1988 to 1994, the Mexico's National Epidemiological Surveillance System reported an epidemic of histoplasmosis owing to its report that showed1,065 cases of the disease, most of which were reported in the states of Veracruz and Oaxaca. Then, in 1995, case notification stopped being compulsory, leading to a decrease in the number ofcases. Currently, in Mexico, the information we have available on this diseasecomes from case series and outbreak reports..$^{7-10}$ These reports have, thereby, generated the necessity of a study being conducted within Mexico's National Institute for Respiratory Diseases with the objective of generating new knowledge on the clinical presentation and main epidemiological characteristics of the histoplasmosis infection in Mexico. Thus, this study aimed to describe the epidemiological, clinical, and paraclinical characteristics of the histoplasmosis infection - which has a wide variety of clinical manifestations - in the population that received care in Mexico's National Institute for Respiratory Diseases.

\section{Materials and methods}

A retrospective observational study was performed. Regarding sample, the inclusion criteria were: Patientsbetween 10 and 99 years of age; having a diagnosis of histoplasmosis confirmed bya positive culture; and having received medical attention at the National Institute of Respiratory Diseasesbetween January 2013 and December 2016. The exclusion criteria were: Patients who did not receive treatment at the National Institute of Respiratory Diseases; those who did not have complete medical records. Clinical and sociodemographic data collection was performed through clinical records reviews of patients who had a positive culture for Histoplasma capsulatum from different clinical samples (i.e. bronchoalveolar lavage, expectoration, bronchial aspiration, biopsy, and bone marrow aspirate). After obtaining theclinical (i.e. coughing, fever, dyspnea, hemoptysis, pleuritic pain, asthenia, adynamia) and sociodemographic (i.e. age, sex, occupation, travel and residence history in endemic areas) data, theywere recorded in an Excel database.Then, statistical analysis was performed using IBM SPSS 21. Results are presented through descriptive statistics; medians with ranges and/or averages with standard deviations were used for quantitative variables, whereasqualitative variables were expressed through frequencies and percentages. We used bivariate analysis for the quantitative variables; for it, patients were divided into two groups: Patients with pulmonary histoplasmosis and patients with its disseminated forms. The corresponding results were compared through an X2 test or Fisher's Exact Test.We usedStudent's T-test and/ or Mann-White $U$ tests for the qualitative variables (i.e. parametric or non-parametric variables). A p-value $<0.05$ was considered as statistically significant. Data were presented along its p-value and odds ratio with a confidence interval of $95 \%$.

\section{Ethical considerations}

This study followedthe establishedethical guidelines for the use of patient information and was approved by the institutional ethical committee at National Institute of Respiratory Diseasesunder the number $\mathrm{C} 31-17$.

\section{Results}

\section{Sociodemographic data}

In total, we found 61 patients with a proven or probable histoplasmosis diagnosis. Theiraverage age was 35.1 years $( \pm 12.4)$. Age distribution is shown in Figure 1. A higher number of cases was observed in male patients, with a male/female ratio of $6: 1$. Moreover, $38 \%(21 / 61)$ of the patients lived in Mexico City, and only 11 (48\%) had a history of travelling to any of the areas in the country that have a high number of reported cases. We observed occupational exposure in 12 patients, and the most common were being construction workers, farmers, gardeners, and miners. However, $80 \%$ of the patients had no occupational risk record.In our 61-patient sample, $80 \%(n=49)$ had a history of immunosuppression. The most common cause of immunosuppression was HIV infection (94\%), followed by diabetes mellitus (2/49), and one patient had a renal transplant and was under immunosuppressive treatment. Amongst HIV cases, 43/46 cases had a CD4 count below 350 at diagnosis. Average CD4 and viral load (CV) were 34 cell/mm3 (interquartile range [IQR] 19-136) and 222,437 copies (IQR 1779-485,752), respectively.

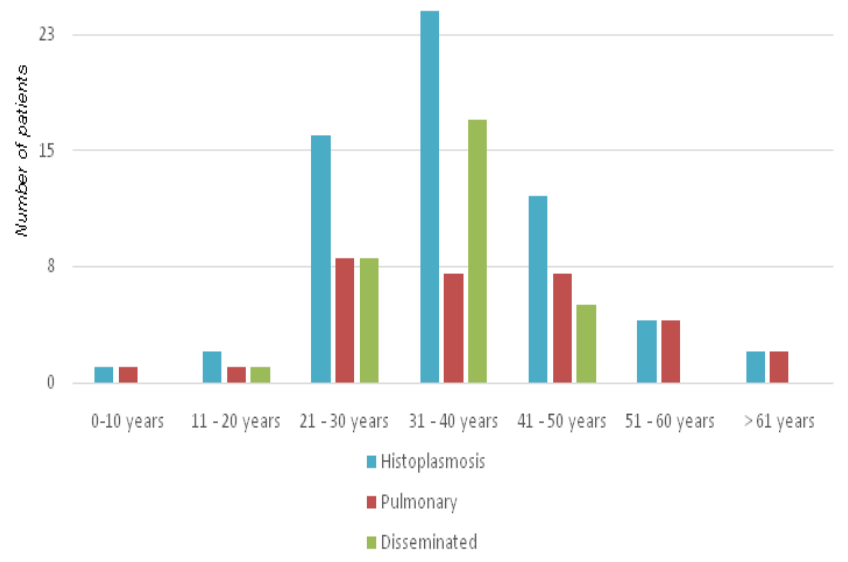

Figure I Patients' age distribution by the diagnosis of different forms of histoplasmosis. 


\section{Clinical data}

In our sample, disseminated histoplasmosis was the most common form (31/61) of the disease. Median symptom duration before diagnosis was 103.9 days (IQR 17-90). This was lower inpatients with pulmonary histoplasmosis, 67.2 (IQR 14-90), compared to patients with a disseminated form, 153.7 (IQR 28-90), but the difference was not statistically significant $(\mathrm{p}=0.32)$. Dyspnea, fever, and diaphoresis were the most common symptoms in patients with a histoplasmosis diagnosis. The frequencies of the main signs and symptoms are shown in Table 1. Hepatomegaly, splenomegaly, and adenopathy were the main clinical signs that were more commonly found in patients with disseminated histoplasmosis compared to those with a pulmonary form, and the differences were statistically significant (Table 1).

\section{Medical imaging data}

The most frequent tomographic sign was the presence of multiple nodular lesions, found in 39/61 patients $(59 \%)$. Particularly, the pulmonary tomography of $2 / 61$ patients $(3 \%)$ reported no structural alteration (Figure 2).

Table I Clinical characteristics of patients with pulmonary and disseminated histoplasmosis

\begin{tabular}{|c|c|c|c|c|c|c|}
\hline Signs and symptoms & Total $(\mathbf{N}=6 I)$ & Pulmonary $(\mathbf{N}=30)$ & Disseminated $(\mathbf{N}=3 \mathrm{I})$ & $\mathbf{P}$ & OR & IC 95\% \\
\hline Dyspnea & $82 \%(50 / 6 I)$ & $86 \%(26 / 30)$ & $77 \%(24 / 3 \mathrm{I})$ & $P=0.34$ & 1.89 & $0.49-7.29$ \\
\hline Cough & $49 \%(30 / 61)$ & $47 \%(14 / 30)$ & $52 \%(16 / 31)$ & $P=0.39$ & 0.76 & $0.27-2.11$ \\
\hline Sputum & $38 \%(23 / 61)$ & $41 \%(12 / 29)$ & $34 \%(|I / 3|)$ & $P=0.46$ & 1.21 & $0.43-3.41$ \\
\hline Hemoptysis & II\% (7/6I) & $21 \%(6 / 29)$ & $3 \%(I / 3 I)$ & $P=0.04$ & 1.92 & $1.26-2.94$ \\
\hline Myalgia & $15 \%(9 / 61)$ & $13 \%(4 / 30)$ & $16 \%(5 / 31)$ & $P=0.52$ & 0.80 & $0.19-3.31$ \\
\hline Fever & $69 \%(42 / 61)$ & $72 \%(22 / 30)$ & $66 \%(20 / 3 I)$ & $P=0.47$ & 1.51 & $0.50-4.51$ \\
\hline Diaphoresis & $56 \%(34 / 6 I)$ & $52 \%(15 / 30)$ & $59 \%(19 / 31)$ & $P=0.36$ & 0.73 & $0.26-2.02$ \\
\hline Weight loss & $54 \%(33 / 61)$ & $53 \%(16 / 30)$ & $55 \%(|7 / 3|)$ & $P=0.90$ & 0.94 & $0.34-2.57$ \\
\hline Hepatomegaly & $26 \%(16 / 6 I)$ & $7 \%(2 / 30)$ & $43 \%(|4 / 3|)$ & $P=0.001$ & 0.87 & $0.18-0.42$ \\
\hline Splenomegaly & $15 \%(9 / 61)$ & $0 \%(0 / 30)$ & $30 \%(09 / 3 I)$ & $P=0.001$ & 2.36 & I.72-3.24 \\
\hline Adenopathy & $30 \%(18 / 61$ & $13 \%(4 / 30)$ & $45 \%(|4 / 3|)$ & $P=0.006$ & 0.18 & $0.53-0.66$ \\
\hline Rales & $62 \%(38 / 6 I)$ & $50 \%(15 / 30)$ & $74 \%(23 / 30)$ & $P=0.05$ & 0.34 & $0.11-1.02$ \\
\hline $\begin{array}{l}\text { Duration of symptoms } \\
\text { (days). }\end{array}$ & $\begin{array}{l}\text { I03.9 (IQR I7-90) } \\
\text { days }\end{array}$ & $\begin{array}{l}67.2 \text { (IQR I4-90) } \\
\text { Days }\end{array}$ & 67.2 (IQR I4-90) days & $P=0.32$ & & \\
\hline
\end{tabular}
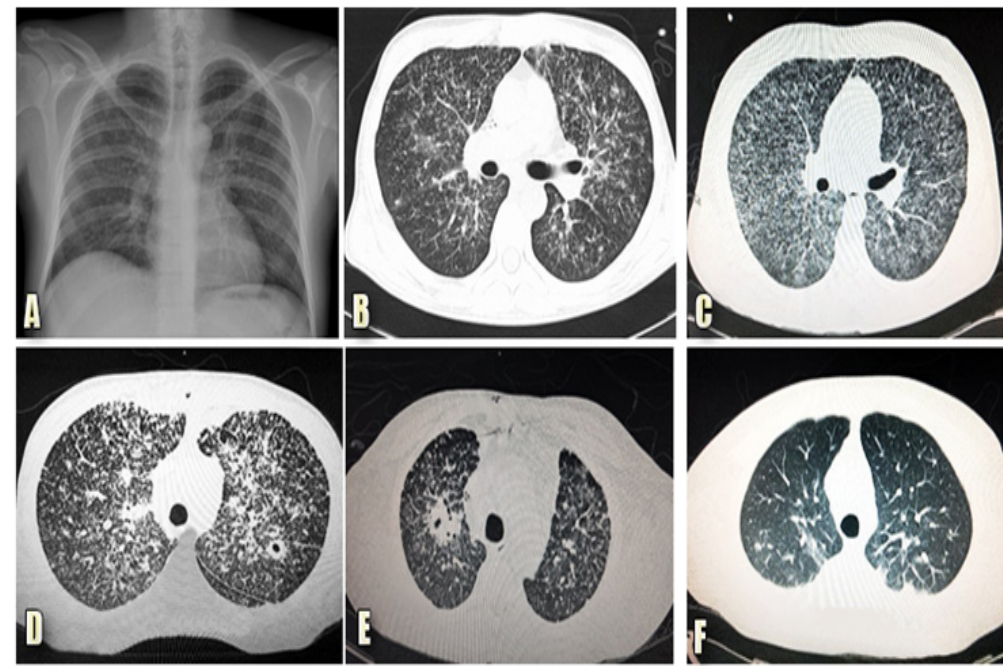

Figure 2 Histoplasma capsulatum manifestations.

The series of images correspond to the following:A. Chest x-ray of an I8-year-old man, without comorbidities, who was exposed to an abandoned building, after which he developed dyspnea and intermittent fever; the image shows a reticulonodular pattern. B. High resolution computed tomography of the same patient; the image shows pleural thickening and random pulmonary nodules of varied sizes. C.High resolution computed tomography of a patient with: HIV infection; non-adherence to antiretroviral treatment; respiratory symptoms for 9 days; evening fever; dyspnea; dry cough; and diagnosed with disseminated histoplasmosis; the image shows randomized micronodules. D. High-resolution computed tomography of a patient with HIV and four months with symptoms; the image shows a pulmonary cavitary nodule. E. High resolution computed tomography of a 44-year-old man from Morelos state with a history of chronic consumption of immunosuppressive therapy; the image shows that there is a micronodular pattern in both lungs and a cavitated nodule in the upper right lobe of the lung. F. High resolution computed tomography of a patient with HIV with: few respiratory symptoms, hepatosplenomegaly, and diagnosed with disseminated histoplasmosis; the image shows some subpleural nodules in the left hemithorax.

Citation: Vargas EB, Tinajero AS, Orozco JAM, et al. Clinical, biochemical, and epidemiological characteristics of patients infected with Histoplasma capsulatum in a non-endemic area of Mexico. J Bacteriol Mycol Open Access. 2020;8(4): I03-I09. DOI: I0.15406/jbmoa.2020.08.0028 I 


\section{Diagnostic methods and laboratory findings}

The principal laboratory findings were: Presence of anemia in 57\% of all patients (35/61), among which the average hemoglobin was 10 $\mathrm{g} / \mathrm{dL} \pm 2.48$; and $78 \%$ of the patients $(45 / 61)$ had an elevated lactic dehydrogenase (LDH), although there were no statistical significant differencesbetween different forms of histoplasmosis. All patients underwent a culture for fungus.In our sample, 92\% (56/61) of the caseshad at least one positive culture for Histoplasma capsulatum growth, and 5 cases reported no growth.Among these 5, 2 were diagnosed through antigen and intracellular yeast presence; 2 only had a positive antigen; and one was diagnosedbyhistopathological findings. In total, 118 cultures (of Sabourad agar) were performed from different clinical samples. Growth of Histoplasma capsulatum was reported in $74 \%(87 / 118)$ of all fungus cultures. In 51 patients, a histopathological study was performed, detecting intracellular yeasts in 25 of them (49\%). The presence of intracellular yeasts was higher in patients with disseminated histoplasmosis $(77 \%)$ than in those with a pulmonary form $(28 \%$; $<0.05)$. A Histoplasma antigen test (i.e. IMMY ALPHA Histoplasma Antigen Enzyme Immunoassay) was performed in 33 patients, with positive results in $67 \%$ of all tests. Moreover, 15 patients underwent a serum galactomannan antigen test, with positive results in $53 \%$ of all tests (Table 2 ).

Table 2 Diagnostic tests comparison in patients with pulmonary and disseminated histoplasmosis

\begin{tabular}{|c|c|c|c|c|c|c|}
\hline Diagnostic test & Total positive & Pulmonary & Disseminated & $\mathbf{P}$ & OR & IC $95 \%$ \\
\hline Microbiological culture & $74 \%(87 / / 18)$ & $67 \%(39 / 58)$ & $81 \%(48 / 59)$ & $P=0.08$ & 0.47 & $0.20-1,10$ \\
\hline \multicolumn{7}{|l|}{ Culture by clinical sample } \\
\hline Culture BAL & $67 \%(26 / 39)$ & $65 \%(17 / 26)$ & $69 \%(9 / 13)$ & $P=0.81$ & 0.84 & $2.01-3.50$ \\
\hline Pulmonary biopsy culture & $76 \%(3 I / 4 I)$ & $67 \%(18 / 27)$ & $93 \%(13 / 14)$ & $P=0.64$ & 0.17 & $0.10-1.36$ \\
\hline Bone marrow culture & $86 \%(18 / 21)$ & $0 \%(0 / 2)$ & $95 \%(18 / 19)$ & $P<0.05$ & 3.0 & $0.60-14.86$ \\
\hline Adenopathy culture & $100 \%(4 / 4)$ & 0 & $100 \%(4 / 4)$ & - & - & - \\
\hline Other respiratory samples & $88 \%(7 / 8)$ & 0 & $88 \%(7 / 8)$ & - & - & - \\
\hline Skin biopsy & $100 \%(1 / 1)$ & - & $100 \%(1 / 1)$ & - & - & - \\
\hline \multicolumn{7}{|l|}{ Histopathology } \\
\hline Histopathology* & $49 \%(25 / 5 I)$ & $28 \%(7 / 27)$ & $77 \%(20 / 26)$ & $P<0.05$ & 3.12 & $1.48-6.56$ \\
\hline \multicolumn{7}{|l|}{ Serology test } \\
\hline & $67 \%(14 / 21)$ & & & & & \\
\hline Urinary antigen & & $67 \%(8 / 12)$ & $67 \%(6 / 9)$ & $P=1.00$ & 1.00 & $0.60-6.25$ \\
\hline Galactomannan & $53 \%(8 / / 5)$ & $29 \%(2 / 7)$ & $75 \%(6 / 8)$ & $P=0.07$ & 0.07 & $0.01-1.31$ \\
\hline
\end{tabular}

BAL: bronchioalveolar lavage

*Periodic acid-Schiff stain (pas) and Grocott-Gomori methenamine silver staining were the special stains used to detect the intracellular yeasts and allow for the histopathological diagnosis

\section{Treatment and outcome}

Only one patient had no record of treatment onset. Out of the 60 treated patients, $71 \%(n=42)$ received initial management with amphotericin B and $29 \%$ withitraconazole. A mortality rate of $7 \%$ was estimated; among the 4 patients that died, all had a disseminated form of histoplasmosis; 3 were male; all were coinfected with HIV; and all had a CD4 count below 200 cell $/ \mathrm{mm}^{3}$, with an average of 19 CD4 cells. Averagely, the deaths occurred within 12 days of hospital admission.

\section{Discussion}

In Mexico, since histoplasmosis stopped being a disease of compulsory notification in 1995 , it has become difficult to accurately estimate the incidence and impact of histoplasmosis on the Mexican population and the Mexican health system. Moreover, histoplasmosis has been reported more frequently in certain groups of patients and there have been an important number of outbreaks. ${ }^{10,11}$ Thus, to the best of our knowledge, the present study could be the first of its kind in Mexico and the first report on the current clinical presentation and epidemiological behavior of histoplasmosis in the country.
This study found a higher presentation of histoplasmosis inmale patients, with a male to female ratio of $6: 1$. This ratio is higher than the 3:1 reported in 1995 by the General Directorate of Epidemiology (DGE) in Mexico, namely, the year in which histoplasmosis stopped being an mandatoryreported disease. ${ }^{7}$ In our sample, the average age at diagnosis was 36.1 years $( \pm 12.4) ; 39 \%$ of the patients were aged from 40-50; and there was a lower frequency in those aged 60 or older. Thesefindings corroboratea report in 1994 and other studies in the Mexican population..$^{7,10}$ However, studies in other samples, such as a systematic revision in India and a case series report in the USA, accounted for an older average age of presentation of histoplasmosis (i.e. $48.6 \pm 8.6$ and 55 years, respectively). ${ }^{12,13}$ Thus, the average age of presentation of histoplasmosis in our Mexican sample was younger than that reported in other countries, and the male to female ratio seems to have increased since the last compulsory report provided in 1995. Correlatively, in Mexico, 32\% of the people living with HIV are between 30 and 40 years old, and the AIDS epidemic has spread mostly among men, with a male to female ratio of almost 4:1. Therefore, given that in our sample $45 / 61$ patients had an HIV infection, this specific morbidity between histoplasmosis and HIV may have been the reason behind the younger age of presentation and male predominance in our country. ${ }^{14,15}$ 
Histoplasmosis is considered endemic in Mexico, with cases reported in 23 of all 32 states; specifically, Morelos, Guerrero, and Veracruz are the states that historically presented higher numbers of reported cases. ${ }^{7}$ In this study, we found cases in 9 states, and $38 \%$ of the patients $(\mathrm{n}=23)$ lived in Mexico City (CDMX), an area of low endemicity. Out of these, 11 patients had a history of traveling to locations that have high endemicity for histoplasmosis in Mexico (Figure 3). Lately, researchers have been observing an increase in the number of cases of histoplasmosis in low endemicity areas; as aforementioned, we found 11 cases that were considered to have been infected in Mexico City.This suggests that histoplasmosis may be extended to regions where the presence of this dimorphic fungus has not been identified and that there is a lack of knowledge on its current distribution in Mexico. ${ }^{12,13}$ Historically, histoplasmosis has been considered as an occupational disease, related with mining, farming, and guano collecting. ${ }^{7}$ However, our study showed that only $20 \%(12 / 61)$ of the cases had an occupational historythat related them to high-risk occupations for histoplasmosis.

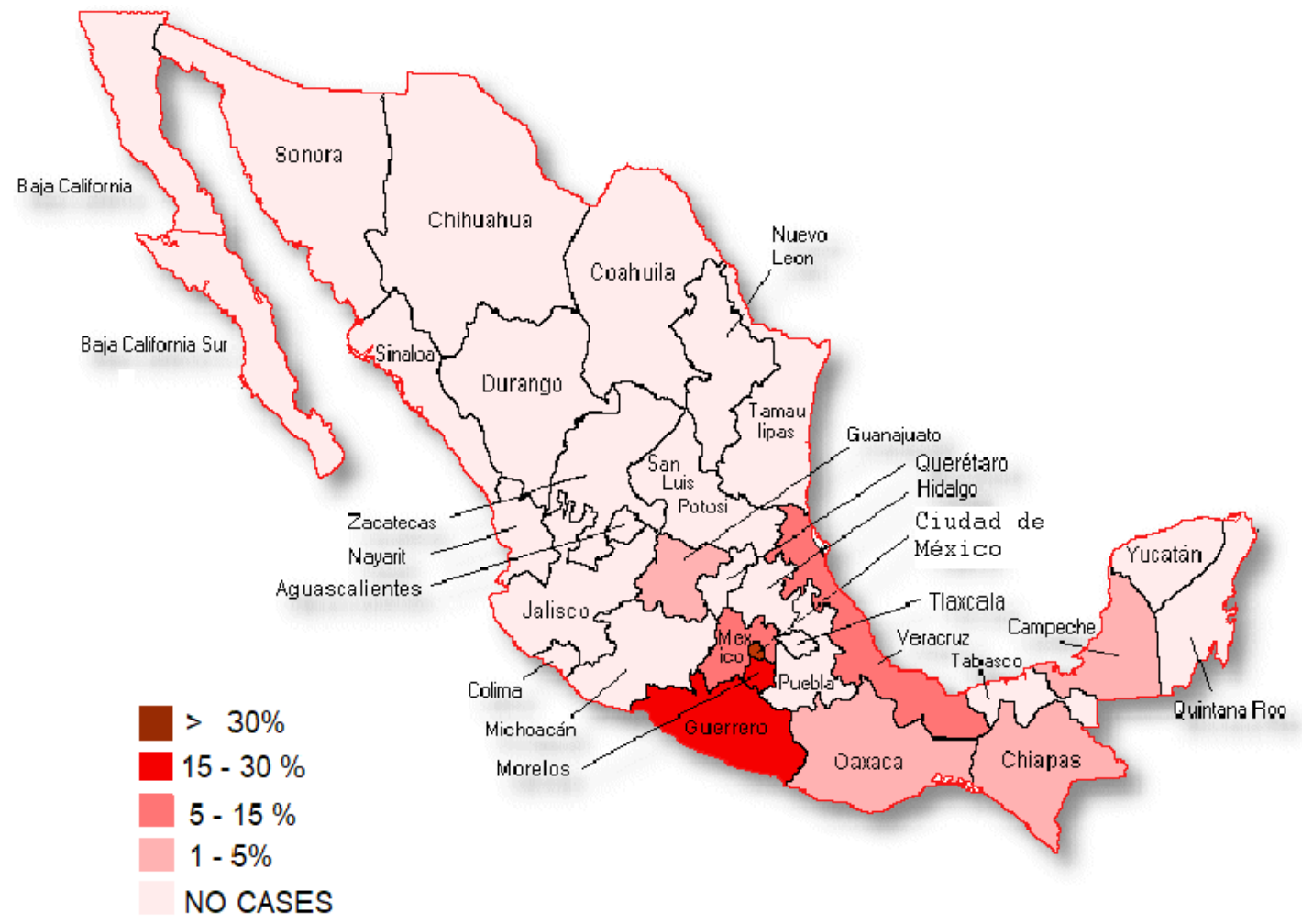

Figure 3 The percentages of reported histoplasmosis cases in México by state. Mexico city, 38\% (23/6I); Guerrero, 16\% (I0/6I); Morelos, I6\% (I0/6I); México, I3\% (8/6I); Veracruz, 8\% (5/6I); Oaxaca, 3\% (2/6I); Campeche, 2\% (I/6I); Chiapas, 2\% (I/6I); Guanajuato, 2\% (I/6I).

Risk factors for developing a histoplasmosis infection are well-known; for example, people with immunosuppression are considered to be at a higher risk of presenting a disseminated form of histoplasmosis. Currently, the main risk factorsfor a disseminated form of histoplasmosis areHIV infectionsandhaving a CD4 cell count below 200. Corroborating, we found that $75 \%$ of the patients presented immunosuppression related to an HIV infection (i.e. a risk factor), and these patients had an average CD4 cell count of $43 \mathrm{cell} / \mathrm{mm}^{3}$. The proportion of patients with an HIV coinfection with histoplasmosis in our study was higher than that reported in other studies in the Mexican population $(65 \%$ in people with an invasive infection of histoplasmosis). Moreover, our results showed that the proportion of people with any comorbidity was larger than that reported in other countries in South America (i.e. Colombia and Brazil) by $6-8 \%{ }^{16}$ One other risk factorof the development or presence of symptoms related to histoplasmosis is the rate of exposure; reportedly, people living in endemic areas have a rate of exposure that ranged from 60$90 \%$. However, in people without immunosuppression, most of these infections are asymptomatic.
In our sample, the most frequent symptoms were dyspnea, fever, and diaphoresis. Compared to our findings, previous studies have reported lower frequencies for dyspnea and general respiratory symptoms. This specific set of symptoms provides us with a possible implication: A patient with fever, dyspnea, a recent diagnosis of HIV, and that eithercame from or has a history of traveling to a high endemicity area could be considered as a candidate to start antifungal treatment, thus helping to speed up diagnosis and treatment of histoplasmosis in the country. ${ }^{13,17}$ Regarding the main radiological signs that we observed, there is, generally, little information in previous literature about this type of clinical data. Still, one study highlighted some information in this regard: Among patients that live in endemic areas, calcified nodules are frequent; among patients with acute infections, consolidation and lymphadenopathy. However, these radiological signs are present in other infectious diseases, so their presence does not make it easier to distinguish between histoplasmosis and other infections. ${ }^{18}$ In the disseminated forms of histoplasmosis, two studies showed that pulmonary lesions are common (i.e. in up to $66 \%$ of cases) andthat nodules with a miliar distribution are the 
most common. ${ }^{15,17}$ Moreover, our results also showed that all patients with pulmonary and disseminated histoplasmosishad tomographic manifestations, and that 94\% (29/32)of the patients that underwent radiographic examinations had radiographic manifestations; once more, our results pointed out a higher proportion than the one found in the literature. ${ }^{15}$ Still, we highlight that, among patients with a localized and disseminated form of histoplasmosis in our sample, multiple nodules were the most frequent radiographic sign. Additionally, 70\% of the patients had two or more tomographic signs. A study pointed out the most frequent laboratory findings related to histoplasmosis in previous literature: LDH elevation, alterations in either alanine aminotransferase (ALT) and aspartate aminotransferase (AST), and the presence of low hemoglobin, platelets, and other cellular lines. ${ }^{18}$ Moreover, Couppié et al. ${ }^{19}$ described that people with dyspnea, LDH elevation, and thrombocytopenia (platelets $<100,000 / \mathrm{mm} 3$ ) had increased mortality ratios. However, we observed only anemia and LDH elevation as the most common laboratory findings in our results; specifically, the median for platelets values was within normal ranges.

To this date, histoplasmosis diagnosis continues to be an important challenge, and the isolation of Histoplasma capsulatum in cultures continues to be the most solid evidence for this infection. However, the results of fungus cultures turnnegative for most patients with its pulmonary form, whereas the same cannot be said about its disseminated forms. In the present study, a positive culture was found in $81 \%$ of the patients with disseminated forms and in $67 \%$ of those with a pulmonary form. Moreover, the identification of yeasts in tissue and fluids allows practitioners to perform a diagnosis in a shorter period of time; yeast-like structures were found in $49 \%$ of the samples analyzed by the pathology service of Mexico's National Institute for Respiratory Diseases. ${ }^{20}$ Additionally, some authors describe cross-positivity with galactomannan tests (i.e. a positivity report of $69 \%$ ), which occurs mostly in patients with disseminated histoplasmosis; ${ }^{21}$ in our study, less than $75 \%$ of the galactomannan tests were positive. In our study, the most prevalent type of treatment was management with Amphotericin B; this result is in accordance with international guidelines that establish Amphotericin B preparations as the first alternative in severe cases, mainly because it allows for a faster recovery process when the diagnosis is confirmed or opportunely suspected. ${ }^{15}$

Histoplasmosis is recognized as an important cause of mortality in immunocompromised populations and in people over 65 years old. ${ }^{7,9}$ Our results showed a mortality rate of $7 \%(4 / 61)$, which is lower than that previously reported. Among the 4 patients that were deceased, all were in an advanced stage of immunosuppression owing to an HIV infection. We believe this may owe to the efficiency of the proposed treatments: Only 3 patients with disseminated histoplasmosis werenot treated with Amphotericin B, and $71 \%$ of the patients received it at the onset of their treatments. This specific - and recommended - treatment could explain the lower mortality ratios we found when compared to prior literature.

\section{Conclusion}

Concluding, our study highlighted the necessity of maintaininghigh clinical suspicion about the differential diagnosis of histoplasmosisbecause it may helpavoid erroneous diagnosis. This is especially true among high risk patients (e.g. HIV patients), and it should be taken into account regardless of occupational hazard history. Our results also showcase the need for early antimycotic therapy interventions to help prevent higher mortality ratios owing to histoplasmosis. In order to achieve this, we recommend for the Mexican governmentto reestablishthe mandatory notification of disseminated histoplasmosis cases, as doing so may help increase the reporting of cases and the awareness of clinicians about this disease.

\section{Acknowledgments}

I thank Cecila Maidé Pinto España for editing the images presented here.

\section{Conflicts of interest}

The authors declare that they have no potential conflict of interests.

\section{References}

1. Deep GS Jr. Histoplasma capsulatum. In: Mandell GL, Dolin R, editors. Mandell, Douglas, and Bennett's principles and practice of infectious diseases. 7th edn. Philadelphia: Churchill Livingstone. Elsevier; 2010:3305-3318.

2. Kauffman CA. Histoplasmosis: A clinical and laboratory update. Clin Microbiol Rev. 2007;20:115-132.

3. Kauffman CA. Histoplasmosis. In: Dismukes WE, Pappas PG, editors. Clinical mycology. New York, NY. Oxford University Press; 2003:285298.

4. Benedict K, Thompson GR, Deresinski S, et al. Mycotic infections acquired outside areas of known endemicity, United States. Emerg Infect Dis. 2015;21:1935-1941.

5. Antinori S. Histoplasma capsulatum: More widespread than previously thought. Am J Trop Med Hyg. 2014;90:982-983.

6. Bahr NC, Antinori S, Wheat LJ, Sarosi GA. Histoplasmosis infections worldwide: Thinking outside of the Ohio River valley. Curr Trop Med Rep. 2015;2:70-80.

7. Vaca Marín MA, Martínez Rivera MA, Flores Estrada JJ. Histoplasmosis in Mexico, historic and epidemiological aspects. Rev Inst Nac Enfermedades Respir. 1998;11:208-215.

8. Nandí-Lozano E, Newton-Sánchez O, Ávila-Figueroa C. Systemic histoplasmosis. Report of five pediatric cases. Bol Med Hosp Infant Mex. 2006;63:47-54.

9. Corcho-Berdugo A, Muñoz-Hernández B, Palma-Cortés G, et al. An unusual outbreak of histoplasmosis in residents of the state of Mexico. Gac Med Mex. 2011;147:377-384.

10. Gaona-Flores VA, Campos-Navarro LA, Cervantes-Tovar RM, et al. The epidemiology of fungemia in an infectious diseases hospital in Mexico city: A 10-year retrospective review. Med Mycol. 2016;54:600-604.

11. Méndez-Tovar LJ, Mejía-Mercado JA, Manzano-Gayosso P, et al. Frequency of invasive fungal infections in a Mexican High-Specialty Hospital. Experience of 21 years. Rev Med Inst Mex Seguro Soc. 2016;54:581-587.

12. Azar MM, Zhang $\mathrm{X}$, Assi $\mathrm{R}$, et al. Clinical and epidemiological characterization of histoplasmosis cases in a nonendemic area, Connecticut, United States. Med Mycol. 2018;56:896-899.

13. Gupta A, Ghosh A, Singh G, et al. A twenty-first-century perspective of disseminated histoplasmosis in India: Literature review and retrospective analysis of published and unpublished cases at a tertiary care hospital in North India. Mycopathologia. 2017;182:1077-1093.

14. Centro Nacional para la Prevención y Control del VIH y el sida. Boletín de Atención Integral de Personas con VIH / Censida. 2017;3(2). 
15. Bonifaz A, Chang P, Moreno K, et al. Disseminated cutaneous histoplasmosis in acquired immunodeficiency syndrome: Report of 23 cases. Clin Exp Dermatol. 2009;34:481-486.

16. Faiolla RC, Coelho MC, Santana R-C, et al. Histoplasmosis in immunocompetent individuals living in an endemic area in the Brazilian Southeast. Rev Soc Bras Med Trop. 2013;46:461-465.

17. Assi MA, Sandid MS, Baddour LM, et al. Systemic histoplasmosis: A 15-year retrospective institutional review of 111 patients. Medicine (Baltimore). 2007;86:162-169.

18. Orlowsky HLP, McWilliams S, Mellnick VM, et al. Imaging spectrum of invasive fungal and fungal-like infections. Radiographics. 2017;37:11191134.
19. Muñoz CO, Cano LE, Ángel G. Detección e identificación de Histoplasma Capsulatum por el laboratorio: De los métodos convencionales a las pruebas moleculares. Infectio. 2010;14:145-148.

20. Wheat LJ. Improvements in diagnosis of histoplasmosis. Expert Opin Biol Ther. 2006;6:1207-1221.

21. Wheat LJ, Hackett E, Durkin M, et al. Histoplasmosis-associated crossreactivity in the BioRad Platelia Aspergillus enzyme immunoassay. Clin Vaccine Immunol. 2007;14:638-640. 